\title{
Prevalence and Associated Factors of Malaria among Febrile Children in Afar Region, Ethiopia: A Health Facility Based Study
}

\author{
Abay Woday ${ }^{1 *}$, Ahmed Mohammed ${ }^{2}$, Abel Gebre ${ }^{1}$, Kusse Urmale ${ }^{3}$
}

\section{OPEN ACCESS}

Citation: Abay Woday, Ahmed Mohammed, Abel Gebre, Kussie Urmalie. Prevalence and Associated Factors of Malaria Mong Febrile Children in Afar Region, Ethiopia: A Health Facility Based Study. Ethiop J Health Sci.2019;29(5):613.doi:http://dx.doi.org/

10.4314/ejhs.v29i5.12

Received: March 26, 2019

Accepted: July 2, 2019

Published: September 1, 2019

Copyright: (c) 2019 Abay Woday, et al.

This is an open access article distributed under the terms of the Creative Commons Attribution License, which permits unrestricted use, distribution, and reproduction in any medium, provided the original author and source are credited.

Funding: Nil

Competing Interests: The authors declare that this manuscript was approved by all authors in its form and that no competing interest exists.

Affiliation and Correspondence:

${ }^{1}$ Department of Public Health, College of Health Science, Samara University, Samara, Ethiopia

${ }^{2}$ Department of Malaria Prevention and Control, Dubti District Health Office, Dubti, Ethiopia

${ }^{3}$ Department of Nursing, College of Health Science, Samara University, Samara, Ethiopia

*Email: abaywoday@yahoo.com
ABSRACT

BACKGROUND: Malaria is a major public health problem in SubSaharan Africa including Ethiopia and it affects children under five and pregnant mothers. Theprevalence of malaria is not well studied though the burden is expected to be high in low lands of Afar region.Therefore,this study attempts to assess the prevalence and associated factors of malaria among under five children in the pastoral community.

METHODS: Cross-sectional study design was employed from April $15^{\text {th }}$ to $15^{\text {th }}$ May 2018 on a sample of 484 under-five children who visited the randomly selected health facilities of Dubti district, Afar region.The study participants were recruited randomly with the basic assumption that their arrival at health facilities is random. The main outcome variable was parasitological confirmed malaria among under five children and it was measured using either microscopy blood film examination. Binary logistic regression model was done to measure the association between confirmed malaria infection and its predictors. Independent variables with p-value less than 0.25 in the bivariate analysis were entered into multivariable logistic regression analysis model. Statistical significance level was declared at p-value less than 0.05 .

RESULTS: The prevalence of malaria among febrile under-five children was 64\% (95\% CI 59.5\%-68.4\%). Of these,more than half (66.5\%) and one-fifth (22. 2\%) of the cases were caused by p.falciparum and p.vivax respectively. The multivariable logistic regression analysis showed thatliving in rural residence [AOR=1.77: 95\% CI 1.10-2.85], having educated mother [AOR=0.406: 95\% CI 0.26-0.63], age of child ([AOR=2.98: 95\% CI 1.41-6.32], sex of child [AOR=1.99: 95\% CI 1.293.08], not sleep under ITN [AOR=9.10: 95\% CI 1.01- 82.58], ITN availability [AOR=2.23: 95\% CI 1.01- 4.89] and history of malaria infection among family members [AOR=9.10: 95\% CI 1.01-82.58] were the independent predictors of malaria infection among under five children.

CONCLUSION: The overall prevalence of malaria among under-five children was high compared to the national prevalence of malaria among the general population. Health care providers (HCPs) should educate parents/guardians regarding proper utilization of ITN at night. In addiotion, they should provide early treatment for febrile family members to halt the transmission of malaria. The regional government should give due attention to supply of ITN and enhance the awareness of the community. Additional longitudinal studies are recommended.

KEYWORDS: Prevalence, Associated factors, Malaria, Febrile Under five children, Ethiopia

DOI: http://dx.doi.org/10.4314/ejhs.v29i5.12 


\section{INTRODUCTION}

Malaria is a public health problem worldwide, and it causes more than 216 million (95\% CI: 196-263 million) cases per year. Of these malaria cases, more than $90 \%$ of are found concentrated in subSaharaAfrican countries including Ethiopia (1). Moreover, in 2016, nearly half million (429 000) deaths were caused by malaria, and more than $92 \%$ of these happened in sub-Sahara African countries (2).

Even though household ownership of at least one ITN was increased from $50 \%$ to $80 \%$ in subSaharan African countries, the proportion of households with sufficient nets (i.e. one net for every two people) remained inadequate (43\%) (1). Furthermore, malaria is still the leading public health problem among sub-Saharan countries including Ethiopia; it predominately affects pregnant mothers and under-five children (3). In Ethiopia, three-fourth of the areas are malaria endemic, and more than two-thirds of its population (more than 55 million people) live in areas of risk for malaria $(4,5)$. According to the 2013 health and health related indicator report of the Ministry of Health $(\mathrm{MOH})$, malaria was one of the ten top leading causes of morbidity (12\%); 2.85 million blood film-confirmed malaria cases were reported from different parts of the country (6).

Globally, regionally as well as nationally different strategies, policies and malaria elimination programs were tried in the last decades (7-10). Over 6.2 million malaria deaths were averted between 2000 and 2015 in subSaharan Africa (11). However, the 2015 target of MDG was not met (reduce the incidence rate by $50 \%$ )(12). Despite the significant decline in the burden of malaria, the disease is still one of the major public health concernins Ethiopia (13). Furthermore, the prevalence of malaria among underfive children ranges between $0.56 \%$ and $53.6 \%$ in the country $(4,14-18)$.

Previous studies conducted across subSaharan African countries to assess the factors associated with malaria endemic identified various factors for the occurrence of malaria endemic among under-five children. The identified factors include sex of the child, age of the child, number of bed nets available for the household members, presence of forest cover, altitude of the residence, household density (family size) and housing conditions $(4,5,15,17,19,20)$.

Afar Region is the most lowland area in Ethiopia, and more than $80 \%$ of its population are at risk for malaria infection, especially under-five children and pregnant women (21). According to the Health Sector Development Plan IV performance (HSDP IV) report, the incidence of malaria in Afar Region was 3,479 cases per 100,000 people (16). Furthermore, according to the 2016 Ethiopian Demographic and Health Survey (EDHS) report, the region has the highest under-five mortality rate compared to other regions (125 deaths per 1000live births) (22). In addition, only $33 \%$ of children under the age of five were sleeping under insecticide treated nets in Ethiopia including Afar Region (23). Therefore, malaria may be responsoble for the highest under-five mortality rate in Afar Region.

Moreover, no study has been conducted todate to address the prevalence and associated factors of malaria among under-five children in Afar Region. Therefore, this study aims to assess the prevalence and associated factors of malaria among under-five children and to fill the identified information gaps in the region.

\section{METHODS AND MATERIALS}

Study setting and participants: Institution based cross sectional study design was employed to assess the prevalence and associated factors of malaria among under-five children getting medical cares in Dubti District public health facilities from $15^{\text {th }}$ April to $15^{\text {th }}$ May 2018. The district is located $588 \mathrm{kms}$ away from the capital city, Addis Ababa. It has a total population of 72,528. Of these, 5,950 are under-five children. The area has 800-1000 meters' altitude above sea level, and it has manmade dam around.The district has four health centers, one referral hospital, fourteen health posts, and three private pharmacies.

All under-five children who presented with fever symptoms and gave blood for microscopic blood film examination or RDT at the randomly selected public health facilities were included in this study. However, children with critically sick care givers/guardians and those who presented to the health facilities without being accompanied by

DOI: http://dx.doi.org/10.4314/ejhs.v29i5.12 
their nearest parents/caretakers were excluded from this study (i.e. children coming with the neighbors or friends).

The sample size was calculated using single population proportion formula with the basic assumptions of $95 \%$ Confidence interval for the Critical value $Z \alpha / 2=1.96$ and $4 \%$ margin of error. A $25 \%$ prevalence (P) of malaria among the general population was taken from a study done in East Oromia [5]. After adding 10\% to compensate the non-responses $(450 \times 0.1=45)$, the final sample size became 495 .

Two health facilities were selected randomly from a total of five health facilities those have functional blood film examination services. The samplee were proportionally allocated into selected health facilities based on malaria case flows they had in the last one-year. The children were assumed to arrive at selected health facilities at random; they were recruited based on their arrival with the inclusion criteria. The data was collected by including all febrile children until the maximum sample size was satisfied. The mothers/caregivers were interviewed about the consistent symptoms of malaria (fever), and final confirmation was done with blood film or RDT results.

Data collection: Standardized, pretested and structured interviewer-administered questionnaire was used to collect data. The questionnaire was developed in English on socio-demographic characteristics, health service related factors, environmental factors, ITN availability and utilization. Two-laboratory technicians and a supervisor from Dubti Hospital were trained for two days on the data collection instrument, interview techniques and recruitment of the study participants.

The tool was pre-tested on $5 \%$ of sample and some amendments were made. The questionnaire was translated into local language (Afar-aaf) and back into English to check for consistency.

Malaria diagnosis was confirmed using the laboratory results done with microscopic blood films prepared from finger-prick blood samples or RDT results collected from malaria suspected children. Experienced laboratory technicians prepared thick and thin blood films that were labeled and air-dried horizontally in a slide tray.
Thin films were fixed with methanol for about 30 seconds, and both thick and thin films were stained with 3\% Giemsa for 20-30 minutes at the study health facilities by using the WHO 2015 standard malaria laboratory procedures guideline (24). The thick films were used for detecting the malaria parasites while the thin films were used for label and for species confirmation. The blood slides were read and then classified qualitatively as either negative, P.falciparum positive, P.vivax positive, or mixed infection. Children with microscopically confirmed malaria infections were treated at the health facilities according to the national malaria diagnosis and the national malaria treatment guidelines.

Data management and analysis: Data entry, data cleaning and data coding were performed using the Epi data version 3.1 and then exported to SPSS version 20.0 foranalysis. The exported data was checked for correlation between independent variables and model fitness was checked using Hosmer-lemeshow model fitness test.

Descriptive statistics was done and the results were presented using texts and frequency tables. Binary logistic regression was done to assess the associations between dependent and independent variables (i.e. sociodemographic factors, health system related factors and environmental factors). Variables with $p$-value $<0.25$ in the bivariate analysis were entered into multivariable logistic regression analysis model. Multivariable logistic regression was done to identify the predictors of malaria among under-five children and to control confounders. Statistical significance level was declared at $\mathrm{P}<0.05$.

Ethical considerations and consent for participants: Ethical clearance was obtained from Samara University, College of Health Science, Department of Public health. A written consent was sought from local authorities and concerned government officials after thoroughly discussing the ultimate purpose and method of the study. Finally, written informed consent was obtained from parents to involve their children in the study. We reassure that anyone who was not willing to participate in the study had full right to refuse and they can stop at any time if started to participate. Blood smears were taken by experienced laboratory technicians under aseptic technique using sterile gloves and disposable sterile lancets. Codes were used to ensure confidentiality of

DOI: http://dx.doi.org/10.4314/ejhs.v28i1.2 
Laboratory results. All cases with history of fever in the preceding three days and/or those who had fever on examination were offered antimalarial treatment as per the national protocol.

\section{RESULTS}

Socio-demographic characters of participants: A total of 484 participants were involved in this study with response rate of $97.7 \%$. The mean age of the children was 28 months $\pm 6.01 \mathrm{SD}$. Of the total participants, $68.0 \%$ resided in rural areas, more than half $(59.5 \%)$ of the children's mothers were illiterate, $83.5 \%$ of the mothers were married, $76 \%$ of the parents were Muslims and nearly half $(42 \%)$ of the mothers were housewives. The mean family size was $3.98 \pm$ 1.43SD (Table 1).

Health service and environmental related factors: Of the total participants, $12 \%$ did not have access to the nearby health facilities, $74.2 \%$ lived around stagnant and open water source environments; $48.6 \%$ of the participants said that there is manmade dam around their residency and $26.5 \%$ of the participants were living within five KMs of the nearby dam. The majority $(81.8 \%)$ of the study participants lived in mud-roof houses (Table 2).

Table 1: Sociodemographic characteristics of participants in Dubti District, Afar Region, Northeast Ethiopia, 2018.

\begin{tabular}{|c|c|}
\hline Predictor variables & Number (\%) \\
\hline \multicolumn{2}{|l|}{ Age of mother (in Years) } \\
\hline $15-24$ & $60(12.4)$ \\
\hline $25-34$ & $249(51.4)$ \\
\hline$\geq 35$ & $175(36.2)$ \\
\hline \multicolumn{2}{|l|}{ Age of the child (in months) } \\
\hline$<12$ & $32(6.6)$ \\
\hline $12-23$ & $176(36.4)$ \\
\hline $24-35$ & $97(20.0)$ \\
\hline $36-47$ & $86(17.8)$ \\
\hline $48-59$ & $93(19.2)$ \\
\hline \multicolumn{2}{|l|}{ Sex of the child } \\
\hline Male & $274(56.6)$ \\
\hline Female & $210(43.4)$ \\
\hline \multicolumn{2}{|l|}{ Residence } \\
\hline Rural & $329(68.0)$ \\
\hline Urban & $155(32.0)$ \\
\hline \multicolumn{2}{|l|}{ Education level of mothers } \\
\hline Literate & $196(40.5)$ \\
\hline Illiterate & $288(59.5)$ \\
\hline \multicolumn{2}{|l|}{ Marital status } \\
\hline Single & $54(11.2)$ \\
\hline Married & $404(83.5)$ \\
\hline Divorced & $11(2.3)$ \\
\hline Widowed & $15(3.1)$ \\
\hline \multicolumn{2}{|l|}{ Religion } \\
\hline Orthodox & $111(22.9)$ \\
\hline Muslim & $368(76.0)$ \\
\hline Others + & $5(1.0)$ \\
\hline \multicolumn{2}{|l|}{$\begin{array}{l}\text { Main occupation of the head } \\
\text { HH }\end{array}$} \\
\hline Housewife & $204(42.1)$ \\
\hline Farmer & $51(10.5)$ \\
\hline Merchant & $78(16.1)$ \\
\hline Government employed & $130(26.9)$ \\
\hline Other++ & $21(4.4)$ \\
\hline
\end{tabular}

Key: "Others+" = Protestant and catholic, "Others++" = daily labor, student, NGO employed

Table 2: Health service, housing conditions and environmental related factors of malaria among

DOI: http://dx.doi.org/10.4314/ejhs.v29i5.12 
under-five children in Dubti District, Northeast Ethiopia, 2018.

\begin{tabular}{cr}
\hline $\begin{array}{c}\text { List of variables } \\
\text { Distance from health facilities }\end{array}$ & $\begin{array}{r}\text { Number } \\
\text { (\%) }\end{array}$ \\
< $5 \mathrm{KM}$ & $125(25.8)$ \\
$>10 \mathrm{KM}$ & $169(34.9)$ \\
Don't know exactly & $57(11.8)$ \\
Open and stagnant water & $133(27.5)$ \\
around residency & \\
Yes & $359(74.2)$ \\
No & $125(25.8)$ \\
Natural or manmade dam & \\
around residency & \\
Yes & $235(48.6)$ \\
No & $249(51.4)$ \\
Distance from the dam & \\
$<5$ Km & $128(26.5)$ \\
$>=5 \mathrm{Km}$ & $262(54.1)$ \\
Don't know & $94(19.4)$ \\
Thatch & $31(6.4)$ \\
Mud & $57(11.8)$ \\
Roof of House & $396(81.8)$ \\
Corrugated iron sheet & $13(2.7)$ \\
Wall of the house & $387(80.0)$ \\
Cement & $84(17.4)$ \\
Wood & $50(10.3)$ \\
Mud & $268(55.4)$ \\
Only one & $166(34.3)$ \\
Tho &
\end{tabular}

Malaria prevalence, ITN ownership and utilization related factors: The overall prevalence of malaria infection among under-five children was $64 \%$ (95\% CI 59.5\% - 68.4\%). More than half $(66.5 \%)$ and one-fifth $(22.2 \%)$ of the cases were caused by plasmodium falciparum and plasmodium vivax respectively. Of the total participants, only below half (40.9\%) had bed nets for their households (Table 3).

Table 3: Malaria infection, ITN ownership and utilization among households of under-five children in Dubti District, Northeast Ethiopia, 2018.

\begin{tabular}{|c|c|}
\hline List of variables & $\begin{array}{l}\text { Number } \\
(\%)\end{array}$ \\
\hline \multicolumn{2}{|l|}{ Family size of the Household } \\
\hline$<4$ & $202(41.7)$ \\
\hline$>=4$ & $282(58.3)$ \\
\hline \multicolumn{2}{|l|}{ Number of U5 children } \\
\hline$<2$ & $339(70.0)$ \\
\hline$>=2$ & $145(30.0)$ \\
\hline \multicolumn{2}{|l|}{$\begin{array}{l}\text { Bed net available for } \\
\text { households }\end{array}$} \\
\hline Yes & $198(40.9)$ \\
\hline No & $284(59.1)$ \\
\hline \multicolumn{2}{|l|}{$\begin{array}{l}\text { ITN utilization (morning } \\
\text { Observation) }\end{array}$} \\
\hline Yes & $161(81.3)$ \\
\hline No & $37(18.7)$ \\
\hline \multicolumn{2}{|l|}{$\begin{array}{l}\text { Who uses the available ITN? } \\
(n=198)\end{array}$} \\
\hline Mothers and children only & $43(21.7)$ \\
\hline Mothers and fathers only & $101(50.9)$ \\
\hline Whole family & $54(27.4)$ \\
\hline \multicolumn{2}{|l|}{$\begin{array}{l}\text { Know methods to prevent } \\
\text { Malaria infection }\end{array}$} \\
\hline Yes & $444(91.7)$ \\
\hline No & $40(8.3)$ \\
\hline \multicolumn{2}{|l|}{ Methods of malaria protection } \\
\hline Sleeping under bed net & $66(13.6)$ \\
\hline Wearing long slave clothes & $247(51.0)$ \\
\hline Spray chemical to houses & $109(22.5)$ \\
\hline Others + & $62(12.8)$ \\
\hline \multicolumn{2}{|l|}{$\begin{array}{l}\text { Laboratory finding of the } \\
\text { child }(n=310)\end{array}$} \\
\hline P.Falciparum & $206(66.5)$ \\
\hline P.Vivax & $72(23.2)$ \\
\hline Mixed infection & $32(10.3)$ \\
\hline \multicolumn{2}{|l|}{ Confirmed malaria infection } \\
\hline Negative & $174(36.0)$ \\
\hline Positive & $310(64.0)$ \\
\hline
\end{tabular}

Key: Others+- use local materials, use different ointments to the exposed body part and use prophylaxis drugs 
Factors associated with malaria among febrile children: After adjustment was made for covariates, children who lived in rural areas had 2 times higher risk of malaria infection compared to those who lived in urban areas $[\mathrm{AOR}=1.77: 95 \%$ CI 1.10-2.85]. The risk of malaria infection increased as the age of children increased $[\mathrm{AOR}=2.98: 95 \%$ CI 1.41-6.32]. Malaria infection was 2-folds higher among female children compared to male children [AOR=1.99: 95\% CI 1.293.08]. Children whose mothers were literate had $59.4 \%$ less risk of malaria compared to those who had illiterate mothers $[\mathrm{AOR}=0.406: 95 \% \mathrm{CI}$ 0.26-0.63]. The risk of malaria infection was 9-folds higher among under-five children who did not sleep under Insecticide Treated Bed Net (ITN) at night compared to those who slept under ITN [AOR=9.10: 95\% CI 1.01- 82.58]. Moreover, the likelihood ratio of malaria infection among under-five children who were living with family members infected with malaria in the last one month was 3-folds higher compared to those who had no family history of malaria infection $[\mathrm{AOR}=2.78: 95 \% \mathrm{CI}$ $1.73,4.45]$ (Table 4).

Table 4: Factors associated with malaria infection among underfive children in Dubti District, Northeast Ethiopia, 2018.

\begin{tabular}{|c|c|c|c|c|c|}
\hline \multirow{2}{*}{\multicolumn{2}{|c|}{ List of variable }} & \multicolumn{2}{|c|}{ Malaria status } & \multirow{2}{*}{$\begin{array}{l}\text { COR } \\
(95 \% \text { CI })\end{array}$} & \multirow{2}{*}{$\begin{array}{l}\text { AOR } \\
(95 \% \text { CI })\end{array}$} \\
\hline & & PositiveN(\%) & Negative N(\%) & & \\
\hline \multicolumn{6}{|l|}{ Residence } \\
\hline & Urban & 197(59.9) & $132(40.1)$ & 1.00 & 1.00 \\
\hline & Rural & $113(72.9)$ & $42(27.1)$ & $1.80(1.18,2.73)$ & $1.77(1.10,2.85)^{*}$ \\
\hline \multicolumn{6}{|c|}{ Sex of the child } \\
\hline & Female & $163(59.5)$ & $111(40.5)$ & $1.58(1.08,2.32)$ & $1.99(1.29,3.08)^{* *}$ \\
\hline & Male & $147(70.0)$ & $63(30.0)$ & 1.00 & 1.00 \\
\hline \multicolumn{6}{|c|}{ Age of child (in Months) } \\
\hline & $<12$ & $17(53.1)$ & $15(46.9)$ & $0.59(0.263,1.34)$ & $1.08(0.44,2.64)$ \\
\hline & $12-23$ & $104(59.1)$ & $72(40.9)$ & $0.75(0.45,1.27)$ & $0.901(0.502,1.62)$ \\
\hline & $24-35$ & $57(58.8)$ & $40(41.2)$ & $0.74(0.41,1.34)$ & $0.72(0.383,1.35)$ \\
\hline & $36-47$ & $71(82.6)$ & $15(17.4)$ & $2.48(1.23,5.01)$ & $2.98(1.41,6.32)^{* *}$ \\
\hline & $48-59$ & $61(65.6)$ & $32(34.4)$ & 1.00 & 1.00 \\
\hline \multicolumn{6}{|c|}{ Education level of the mother } \\
\hline & Illiterate & $142(72.4)$ & $54(27.6)$ & 1.00 & 1.00 \\
\hline & Literate & $168(58.3)$ & $120(41.7)$ & $0.532(0.36,0.787)$ & $0.406(0.26,0.63)^{* * *}$ \\
\hline \multicolumn{6}{|c|}{ ITN utilization (morning observation) } \\
\hline & Yes & $89(55.3)$ & $72(44.7)$ & 1.00 & 1.00 \\
\hline & No & $221(68.4)$ & $102(31.6)$ & $1.75(1.18,2.58)$ & $9.10(1.01,82.58)^{*}$ \\
\hline \multicolumn{6}{|c|}{ Number of ITN for family } \\
\hline & Zero & $219(67.6)$ & $105(32.4)$ & $2.28(1.21,4.31)$ & $2.23(1.01,4.89)^{*}$ \\
\hline & $1-2$ & $70(60.3)$ & $46(39.7)$ & $1.66(0.83,3.35)$ & $0.35(0.038,3.25)$ \\
\hline & $>=3$ & $21(47.7)$ & $23(52.3)$ & 1.00 & 1.00 \\
\hline \multicolumn{6}{|c|}{ Family size (including extended families) } \\
\hline & $<4$ & $118(58.4)$ & $84(41.6)$ & 1.00 & \\
\hline & $>=4$ & $192(68.1)$ & $90(31.9)$ & $1.52(1.04,2.21)$ & \\
\hline \multicolumn{6}{|c|}{ malaria infection among family In the last one months } \\
\hline & Yes & $256(69.0)$ & $115(31.0)$ & $2.43(1.58,3.74)$ & $2.78(1.73,4.45)^{* * *}$ \\
\hline & No & $54(47.8)$ & $59(52.2)$ & 1.00 & 1.00 \\
\hline \multicolumn{6}{|c|}{ Present of stagnant water around } \\
\hline & Yes & $245(68.2)$ & $114(31.8)$ & $1.98(1.31,3.01)$ & \\
\hline & No & $65(52.0)$ & $60(48.0)$ & 1.00 & \\
\hline \multicolumn{6}{|c|}{ Distance from dam } \\
\hline & $<5 \mathrm{Km}$ & $86(67.2)$ & $42(32.8)$ & $1.72(0.99,2.98)$ & \\
\hline & $>=5 \mathrm{Km}$ & $224(62.9)$ & $132(37.1)$ & 1.00 & \\
\hline \multicolumn{6}{|c|}{ Distance from the nearby health facilities } \\
\hline & $<5 \mathrm{Km}$ & $69(55.2)$ & $56(44.8)$ & 1.00 & \\
\hline & $5-10 \mathrm{Km}$ & $108(63.9)$ & $61(36.1)$ & $1.43(0.89,2.30)$ & \\
\hline & $>10 \mathrm{Km}$ & $133(70.0)$ & $57(30.0)$ & $2.08(1.05,4.09)$ & \\
\hline
\end{tabular}

Key: $\mathrm{COR}=$ Crude Odds Ratio, $\mathrm{AOR}=$ Adjusted Odds Ratio, ${ }^{*}=\mathrm{P}<0.05, * *=\mathrm{P}<0.01, * * *=\mathrm{P}<0.001$

DOI: http://dx.doi.org/10.4314/ejhs.v29i5.12 


\section{DISCUSSION}

Institution based cross sectional study design was employed to assess the prevalence and associated factors of malaria among febrile under-five children. The overall prevalence of malaria infection among under-five children was comparable to the general population. After adjusting for covariates, residence, education level of head of household, age of child, sex of child, ITN utilization, number of ITN available and history of malaria infection among family members in the last one month were the main predictors of malaria among febrile under-five children.

The overall prevalence of malaria infection among under-five children was $64 \%$ which is higher than a study done in BennaTsemay District, Southern Ethiopia (6.1\%) (17), a study conducted in Eritrea (2.2\%) (25), a study done in Jigjiga, Ethiopia (2.8\%) (15),a study done in Dilla District, Ethiopia (16\%) (18), a study done in East Shewa Zone of Oromia Region, Ethiopia (25\%) (5), a study done in East Shewa Zone of Oromia, Ethiopia (20.5\%) (4), a study done in Zambia $(54.3 \%)(26)$, and a study done in Djibouti $(6.9 \%)$ (27). However, the prevalence in this study is lower than the study done in sub-Saharan African countries (64.5\%) (28), a study done in Burkina Faso (68\%) (29) and a study done in Tanzania $(78.91 \%)(30)$. The difference could be justified by the fact that the present study was conducted in the lowest altitude of Ethiopia which is malaria endemic compared to the settings of the previous study settings. There might be cut-point of children's age differences, sociocultural discrepancy and topographic differences between study settings.

Children who lived in rural areas had two times higher risk of malaria infection compared to those who lived in urban settings. This finding is in line with a study done in East Shewa Zone of Oromia Region (5), a study done in Burkina Faso (29) and a study done in Tanzania (30). This might be explained by the fact that children living in the rural community do not have access to available malaria protective methods including ITN use during night time and they do not have access to other housing and clothing methods. Moreover, children in rural community spend more time outdoor compared to children in the urban community. Because of these reasons, they are more at risk for malaria infection.

This study revealed that as the age of children increased, risk of malaria infection increased by three folds. This finding is in line with a study done in Malawi (31) and a study conducted in Uganda to assess the risk factors associated with malaria infection among under-five children (19), a study done in Zambia [45] and a study done in Awassa District, Ethiopia (32). This could be justified by this is the immediate time ( $4-5$ years of age), in which children participate to look after the cattle. Therefore, as the age of the children increases, they may stay out-door by looking after the catle, in which they can be more prone for malaria infection.

Malaria infection among female children was 2 times higher compared to male children. However, this finding is not supported by the study done in Gilgel Gibe Health and Demographic surveillance system, Ethiopia (33). Although males are more prone to malaria because of their outdoor spending in Ethiopia, the discrepancy could be explained by the existence of gender-based discrimination practice in pastoral rural community, such as unequal food and other care provision which lead to impaired immunity of the children.

The likelihood of malaria infection among children who had educated mothers was less likely by $59.4 \%$ compared to those who had illiterate parents. This is in line with the study conducted in Uganda to assess the risk factors associated with malaria infection among under-five children (19), a study done in Madagascar (34) and a study done in Malawi (35). This could be justified by increased awareness of the mothers to proper use of the available ITN during sleeping which in turn increases the protection of malaria among children and the whole family, too. Moreover, when the education level of mothers increases, their knowledge about the available health services and feeding practice will also increase. Because of these reasons, children who had educated mothers had less risk of malaria infection.

DOI: http://dx.doi.org/10.4314/ejhs.v28i1.2 
Sleeping under insecticide treated bed net at night is the main stay of protection mechanism from malaria infection in lowland areas. This study revealed that under-five children who were not sleeping under ITN had nine-fold higher risk of malaria infection compared to those who were sleeping under ITN. This finding is supported by a study done in Jimma Town, Southwest Ethiopia (36), a study done in Malawi (31), and a community based RCT study done in Southern Ethiopia (37). The presence of active malaria infection among family members or neighborhoods can create the opportunity of infecting the apparently healthy under five children. Therefore, children and other family members should sleep under ITN at night to halt the transmission of malaria.

In this study, under-five children who were among households who did not have any ITN for the family members were two times more likely to be have malaria infection compared to those who had ITN for their families. This finding is similar with a study done in Northeast Ethiopia (4), a study done in Dilla District, Ethiopia (18), a study done in Oromia Region (5), and a study done to assess the malaria related factors among underfive children in rural Ethiopia (38). This could be explained by the fact that children from households who did not have bed nets are at high risk for malaria compared to those have have bed nets.

The presence of malaria infection among family members or around them can be used as good opportunity to infect under-five children and other apparently healthy family members. In this study, the likelihood of malaria infection among under-five children who had family members infected with malaria in the last one month was three-folds higher compared to those who had no family history of malaria infection. This might be justified by the fact that children from family members infected by malaria are more likely to be infected by malaria. This is because of the mosquito had access to feed on infected blood and transmitted the malaria to apparently healthy children living with infected family members. However, distance from health facilities, living around stagnant water and family size were not significantly associated with malaria infection, after adjustment was made for selected covariates.

Although morning observational checklist was used to observe usage of the available bed nets, the study was not supplemented with qualitative methods to explore the possible barriers which hindered households' use of available bed nets at night time. Moreover, the study shares the limitations of cross sectional study designs.

In conclusion, this study revealed that the overall prevalence of malaria among under-five children was higher than the regional health bureau reports. Therefore, the findings of this study can help the region to intensively work on the distribution of ITN and other malaria prevention and control modalities to eliminate burden of malaria among under-five children.

In addition, healthcare providers should provide effective treatment for newly malaria infected patients to halt the transmission. Health education should be given for the community about proper utilization of the available bed nets to alter the transmission of malaria.

The district should supply adequate ITNs for the community. It should monitor the proper utilization of the distributed bed nets in the community. Additional longitudinal studies are recommended.

\section{REFERENCES}

1. WHO. World malria report 2017. Geneva, switzerland, 2017.

2. WHO. world-malaria-report-2016. Geneva, switzerland, 2016.

3. Kassam R, Sekiwunga R, MacLeod D, Tembe $\mathrm{J}$, Liow E. Patterns of treatment-seeking behaviors among caregivers of febrile young children: a Ugandan multiple case study. $B M C$ Public Health 2016; 16:160.

4. Hajia. Y, Fogartyb. AW, Deressa. W. Prevalence and associated factors of malaria among febrile children in Ethiopia: A crosssectional health facility-based study. Acta Tropica 2016; 155:63-70.

5. Tadesse. F, Fogarty. AW, Deressa. W. Prevalence and associated risk factors of malaria among adults in East Shewa Zone of Oromia Regional State, Ethiopia: a cross-

DOI: http://x.doi.org/10.4314/ejhs.v29i5.12 
sectional study. BMC Public Health 2018; $18: 25$.

6. FMOH. Health and Health Related Indicators. FMOH. vol. 2, 2013.

7. FMOH. Health Sector Development Program IV (2010/11 - 2014/15) final draft. Addis Ababa, Ethiopia, 2010.

8. FMOH. Health Sector Transformation Plan (HSTP 2016-2020). Addis Ababa, Ethiopia, 2015.

9. United Nations. The Millennium Development Goals; 1990-2015.

10. United Nations: Transforming our world: the 2030 Agenda for Sustainable Development, 2015.

11. United Nations. The Millenium Development Goals 2015 report. New york, 2015.

12. FMoFED. Assessing progress towards the Millenium Development Goals: Ethiopia MDGs REPORT 2012. Addis Ababa, Ethiopia, 2012.

13. Jima. D, Wondabeku. M, Alemu. A, et al. Analysis of malaria surveillance data in Ethiopia: what can be learned from the Integrated Disease Surveillance and Response System? Malaria Journal 2012; 11:330.

14. Ashton RA, Kefyalew T, Tesfaye G, et al. School-based surveys of malaria in Oromia Regional State, Ethiopia: a rapid survey method for malaria in low transmission settings. Malar J 2011; 10:25.

15. Ayalew. S. The Prevalence of Malaria and the Associated Risk Factors in Jiga area, Northwest Ethiopia. Addis Ababa University Elibrary, 2014.

16. FMOH. HealtH Sector Development programme Iv annual performance report (2013/14). Addis Ababa, Ethiopia, 2014.

17. Debo. GW, Kassa. DH. Prevalence of malaria and associated factors in Benna Tsemay district of pastoralist community, Southern Ethiopia. Tropical Diseases, Travel Medicine and Vaccines 2016; 2:16.

18. Molla. E, Ayele. B. Prevalence of Malaria and Associated Factors in Dilla Town and the Surrounding Rural Areas, Gedeo Zone, Southern Ethiopia. J Bacteriol Parasitol 2015; 6:5.
19. Roberts D, Matthews G: Risk factors of malaria in children under the age of five years old in Uganda. Malar J 2016; 15:246.

20. Tassew. A, Hopkins. R, Deressa. W. Factors influencing the ownership and utilization of long-lasting insecticidal nets for malaria prevention in Ethiopia. Malaria Journal 2017; $16: 262$.

21. CSA. third Population and Housing Census, Addis Ababa, Ethiopia, 2007.

22. CSA. ETHIOPIA Demographic and Health Survey 2016. Addis Ababa, Ethiopia, 2017.

23. Afar Regional Health Bureau. Afar Regional Health Bureau 2017 malaria report. unpublished report, Semera, Ethiopia, 2017.

24. WHO. Methods Manual: Microscopy for the detection, identification and quantification of malaria parasites on stained thick and thin blood films in research settings. 2015.

25. David M. Sintasath Tg, Matthew Lynch, Eckhard Kleinau, et al. Malaria Prevalence And Associated Risk Factors In Eritrea. Am J Trop Med Hyg, 2005; 72:6:pp. 682-687.

26. Sitali L, Chipeta J, Miller JM, et al. Patterns of mixed Plasmodium species infections among children six years and under in selected malaria hyper-endemic communities of Zambia: population-based survey observations. BMC Infect Dis, 2015; 15:204.

27. Noor AM, Mohamed MB, Mugyenyi CK, et al: Establishing the extent of malaria transmission and challenges facing preelimination in the Republic of Djibouti. $B M C$ Infect Dis 2011; 11:121.

28. Shah JA, Emina JB, Eckert E, Ye Y. Prompt access to effective malaria treatment among children under five in sub-Saharan Africa: a multi-country analysis of national household survey data. Malar J 2015; 14:329.

29. Sekou Samadoulougou MM-G, Fati KirakoyaSamadoulougou, Mathilde De Keukeleire, Marcia C Castro and Annie Robert. Multilevel and geo-statistical modeling of malaria risk in children of Burkina Faso. Parasites \& Vectors: 2014; 7:350.

30. Adinan J, Damian DJ, Msuya SE. Factors Associated with Testing and Prompt Use of Recommended Antimalarials following Malaria Diagnosis: A Secondary Analysis of

DOI: http://dx.doi.org/10.4314/ejhs.v28i1.2 
2011-12 Tanzania HIV and Malaria Indicator Survey Data. PLoS One 2015; 10:7:e0132964.

31. Zgambo. M, Mbakaya. BC, Kalembo. FW Prevalence and factors associated with malaria parasitaemia in children under the age of five years in Malawi: A comparison study of the 2012 and 2014 Malaria Indicator Surveys (MISs). PloS One 2017; 12:4.

32. Alemu. G. Prevalence of Malaria and its influencing factors in Awassa District, Southern Ethiopia. Addis Ababa University elibrary, 2006.

33. Sena. L, Deressa. W, Ali. A. Dynamics of Plasmodium falciparium and Plasmodium vivax in a micro-ecological setting, Southwest Ethiopia: effects of altitude and proximity to a dam. BMC Infectious Diseases 2014; 14:625.

34. Clouston SA, Yukich J, Anglewicz P. Social inequalities in malaria knowledge, prevention and prevalence among children under 5 years old and women aged 15-49 in Madagascar. Malar J 2015; 13 Suppl 1:499.

35. Chitunhu S, Musenge E. Direct and indirect determinants of childhood malaria morbidity in Malawi: a survey cross-sectional analysis based on malaria indicator survey data for 2012. Malar J 2015; 14:265.

36. Alemu A, Tsegaye W, Golassa L, Abebe G. Urban malaria and associated risk factors in Jimma town, south-west Ethiopia. Malar J $2011 ; 10: 173$

37. Deressa. W, Yihdego. YY, Kebede. Z, Batisso. E, Tekalegne. A, Dagne. GA. Effect of combining mosquito repellent and insecticide treated net on malaria prevalence in Southern Ethiopia: a cluster-randomised trial. Parasites \& Vectors 2014; 7:132.

38. Deressa. W, Ali. A. Malaria-related perceptions and practices of women with children under the age of five years in rural Ethiopia. BMC Public Health 2009; 9:259. 